\title{
The Design Of A Clicker Game for Text Labelling
}

\author{
Chris Madge \\ EECS \\ Richard Bartle \\ Jon Chamberlain \\ Udo Kruschwitz \\ CSEE \\ CSEE \\ CSEE \\ Massimo Poesio \\ EECS \\ Queen Mary Univ. Of London
United Kingdom \\ University Of Essex University Of Essex University Of Esse. \\ United Kingdom United Kingdom United Kingdom \\ rabartle@essex.ac.uk jchamb@essex.ac.uk udo@essex.ac.uk \\ Kingdom \\ c.j.madge@qmul.ac.uk \\ m.poesio@qmul.ac.uk
}

rabartle@essex.ac.uk jchamb@essex.ac.uk u

\begin{abstract}
udo@ess.uk
\end{abstract}
\begin{abstract}
Games for text annotation / labelling are becoming more common, but it's difficult to find a mechanics that fits. In this work we discuss a clicker game that can support text annotation. We believe this type of game is uniquely suited to addressing some of the challenges faced by games featuring text annotation as a core task.

Index Terms-game design, clicker games, games with a purpose
\end{abstract}

\section{INTRODUCTION}

Text annotation is now commonplace in games. At first this was motivated by the application of the Games With A Purpose (GWAP) paradigm to language resourcing [1], [1][6]. GWAP creators explored various approaches, from the not so game-like gamification [1], [3] or a multiple choice question format [4], through to mimicking an existing game design and wrapping that around the annotation task to deliver a more game-like experience [5], [6] or having the player annotate to earn upgrades in a game [7]. But despite a number of attempts, GWAPs for text annotation have failed to achieve the scale (in number of players and/or amount of annotated data) of GWAPs in other domains [8], [9] or truly embrace the GWAP design principles of contribution as a byproduct of play [1], [3], as per the original definition [10]. This is largely because text annotation is not an easy fit into games. Thus, text annotation GWAP creators tend to borrow unsuitable game mechanics (dubbed "orthogonal game mechanics" in [11]). However, such tasks have also been considered to be GWAP's greatest opportunity as unlike crowdsourcing methods, GWAPs can [11], and do make use of game-like training opportunities such as tutorials [1], [3].

In this work, we have been drawing inspiration from socalled clicker games. The first clicker game was a work satirical of 'Ville games [12], but they have since evolved into a popular sub-genre [13]. Distilling Social Network Game (SNG) patterns into small, simple games, inexpensive, yet infinite games with high ludic efficiency [14] make them uniquely suited to gamification problems [15]. Others have pointed out that the broader category of idle games could have benefits to crowdsourcing, or applications that typically benefit from gamification [16]. However, to our knowledge, our work is the first example of an attempt at such a game.

\section{WordClicker}

\section{A. Story}

The story takes inspiration from the game "Cookie Clicker", in which the player plays the role of someone with a cookie business. In WordClicker the player plays the role of a cake shop owner. Their job is to produce cakes by discovering the relevant ingredients, and the business by choosing when and how to reinvest their profits in expanding their business (buying bakeries), improving production (ovens) or increasing efficiency (improved equipment/the amount of ingredients found). Unlike a normal cake shop, the owner/player is responsible for finding individual ingredients. The player must identify words that match an ingredient or part of speech to collect them.

\section{B. Gameplay}

The game is a clicker game, with two types of resources (cakes and ingredients) and a single virtual currency. The main player action is word labelling - clicking on tokens that match a part of speech (i.e. proper noun; pronoun; noun; verb; adjective). Those parts of speech are mapped to the ingredients resource. The player can select an ingredients jar, then select one of more tokens that match the part of speech. Correctly identifying that part of speech will accumulate more ingredients in the jar. The production of the cakes is automatic. The ingredients go down over time in line with the speed of cake production. If ingredients are available, they are automatically added to the cakes as they are produced. Collectively, the cakes and the currently available ingredients transition into the virtual currency. The more ingredients are available, the nicer the cakes are, and the more they are worth. An optimal strategy would see the player prioritising identifying ingredients based on maintaining their availability at all times, in turn maximising profits.

The player is encouraged to reinvest their profits from the cake in purchasing items from the shop. These items are hidden until the player has almost enough virtual currency to first afford them. A progress bar shows how close the player is to be able to afford an item. These cake production related items come in three types. The first and most common type enable the player to increase the speed of cake production. The second type allow the player to extract more ingredients for each correct token they find. The third allows the player to 
apply a new type of label, also raising the maximum potential value of their cakes. The first two effectively enables the player to govern the overall speed of their interaction with the game (investing in higher cake production speeds the game up/investing in higher resource utilization slows it back down), all whilst exponentially increasing their reward. The third allows for a self-paced progression into new word labels.

If the player makes a mistake, one of their purchases is damaged. Once the purchase becomes $100 \%$ damaged, it is removed from the players inventory, and they cease to benefit from the in-game advantage that it offered.

In terms of the core game loop this is:

- Action select tokens matching part of speech tags

- Wait automated cake production takes available ingredients - makes cakes

- Reward ingredients combined with cakes become currency

- Upgrade virtual currency is used to purchase upgrades

The game features a short interactive tutorial (Figure 1). This highlights where the player can find information in the interface, then walks them through changing ingredients, selecting tokens, and making their first purchase.

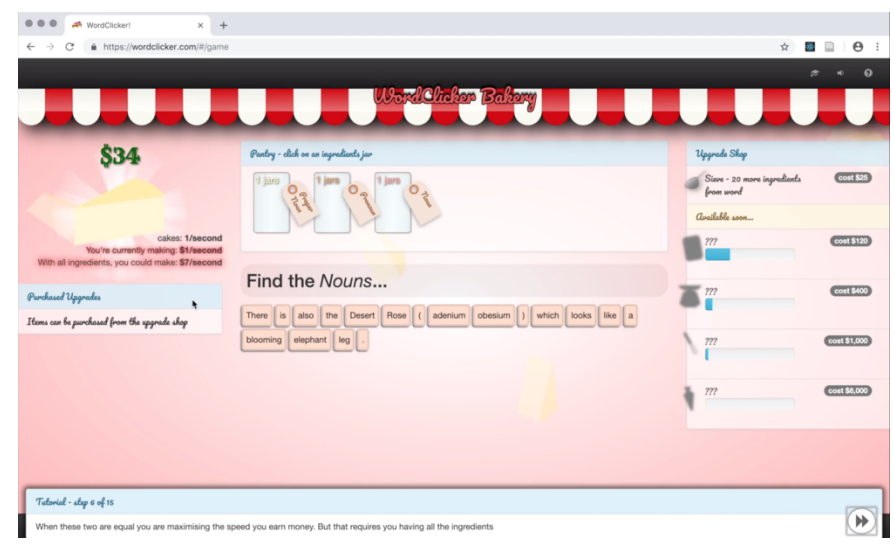

Fig. 1. WordClicker- Tutorial

\section{Art}

From the outset, the game is styled like a cake shop front with a red and white awning, and a noticeboard that introduces the game (Figure 2). This is theme is continued into the game itself.

Ingredients and part of speech tags are colour coded to communicate the link between labels/ingredients and game elements with players. Each ingredient has a colour, this is used in the ingredients jar and on correctly labelled tokens. When an ingredients jar is selected, the background colour of the game changes to that colour to reflect the current selection.

The cake pictures, both the cake in the foreground and those falling in the background, reflect the players currently available ingredients. All the combinations of cakes available, depending on the availability of ingredients, are shown in Table I.

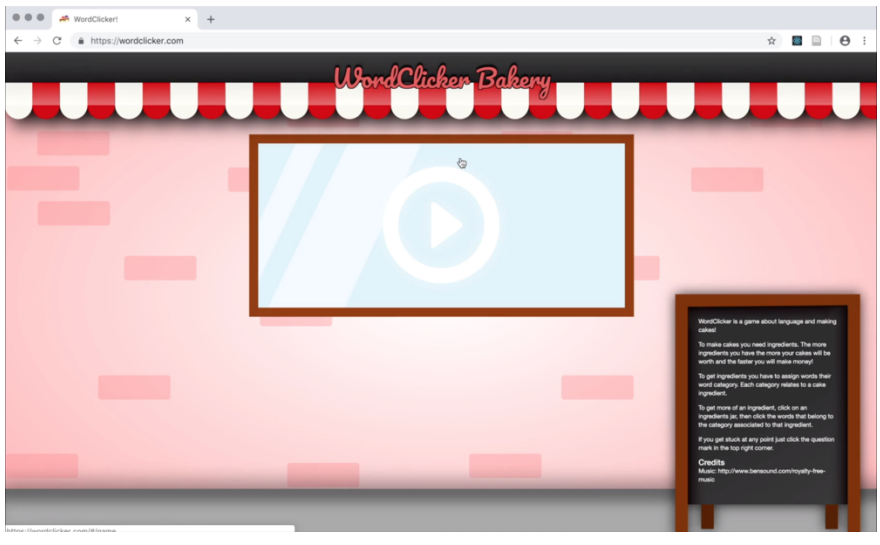

Fig. 2. WordClicker- Home

The game is predominately $2 \mathrm{D}$, making use of a minimalistic, vector drawn, cel shading graphics style reminiscent of early Social Network Games. However, there are small 3D elements to add emphasis. For example, the instructions are shown in the form of a 3D cookbook that opens with an animation (Figure 3). The cakes falling in the background are displayed at various sizes and fall at various speeds to give the illusion of depth.

Three fonts have been selected. Two are heavily stylised serif fonts. The first gives the appearance of a very old cash register, and is used to display the virtual currency. The second has a restaurant menu feel to it, and is used throughout the in game displays. The third is a sans-serif font that is used only in instructional or help settings for enhanced readability.

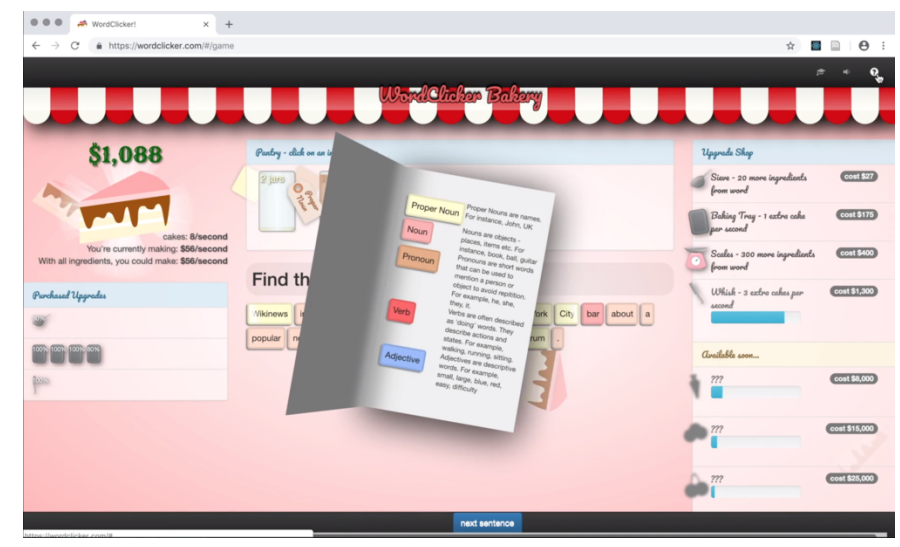

Fig. 3. WordClicker- Instructions

Animations are used to communicate the relationship between resources and the virtual currency. A correctly identified token shows an animation of a colour coded square travelling from the token to its ingredients jar, and from the ingredients jar to the cake.

We give the illusion of a three dimensional background by having various size cakes falling at different speeds. The ingredients present on the cakes changes depending on the ingredients the player has available. 


\begin{tabular}{|c|c|c|c|c|c|}
\hline PN & N & P & A & V & cake \\
\hline$x$ & $x$ & $x$ & $x$ & $x$ & \\
\hline$\checkmark$ & $x$ & $x$ & $x$ & $x$ & \\
\hline$x$ & $\checkmark$ & $x$ & $x$ & $x$ & \\
\hline$x$ & $x$ & $\checkmark$ & $x$ & $x$ & - \\
\hline$x$ & $x$ & $x$ & $\checkmark$ & $x$ & $\infty$ \\
\hline$x$ & $x$ & $x$ & $x$ & $\checkmark$ & $\infty$ \\
\hline$x$ & $\checkmark$ & $x$ & $\checkmark$ & $x$ & $\infty$ \\
\hline$x$ & $\checkmark$ & $x$ & $\checkmark$ & $\checkmark$ & $\infty$ \\
\hline$x$ & $\checkmark$ & $x$ & $x$ & $\checkmark$ & $\infty$ \\
\hline$x$ & $x$ & $x$ & $\checkmark$ & $x$ & $\infty$ \\
\hline
\end{tabular}

\begin{tabular}{|c|c|c|c|c|c|}
\hline PN & $\mathrm{N}$ & $\mathrm{P}$ & A & V & cake \\
\hline$x$ & $x$ & $\checkmark$ & $\checkmark$ & $x$ & ला \\
\hline$x$ & $\checkmark$ & $\checkmark$ & $\checkmark$ & $x$ & ला" \\
\hline$x$ & $\checkmark$ & $\checkmark$ & $x$ & $x$ & $\mathrm{~mm}$ \\
\hline$x$ & $x$ & $\checkmark$ & $x$ & $\checkmark$ & लाm \\
\hline$x$ & $x$ & $\checkmark$ & $\checkmark$ & $\checkmark$ & $\frac{\infty \infty}{\omega \pi}$ \\
\hline$x$ & $\checkmark$ & $\checkmark$ & $\checkmark$ & $\checkmark$ & का \\
\hline$x$ & $\checkmark$ & $\checkmark$ & $\checkmark$ & $\checkmark$ & $\frac{\infty}{\omega \pi}$ \\
\hline$\checkmark$ & $x$ & $x$ & $\checkmark$ & $x$ & $\infty$ \\
\hline$\checkmark$ & $\checkmark$ & $x$ & $\checkmark$ & $x$ & $\omega$ \\
\hline$\checkmark$ & $\checkmark$ & $x$ & $\checkmark$ & $\checkmark$ & $\infty$ \\
\hline
\end{tabular}

TABLE

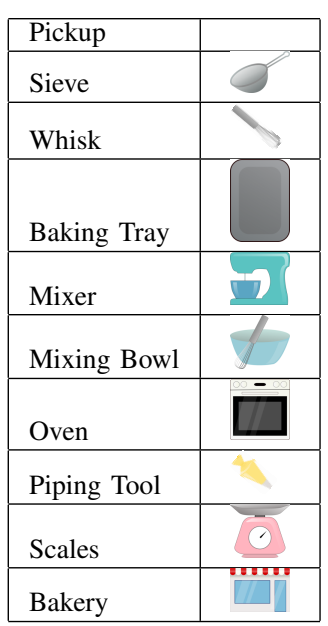

CAKes For Parts-of-Speech/Ingredients (Proper-noun: PN; Noun: N; Pronoun: P; Adjective: A; Verb: V) AND Examples Of Pickups

\section{User Interface and Game Controls}

A panel like interface is used to easily support the responsive design. The interface operates at a variety of screen sizes, including mobile. When viewed on a mobile, the primary interface panels collapse into a vertical view. The secondary panels are available as modal interfaces via buttons that then show in the navigation bar. All interactive elements are large buttons designed to be suitable for touch screen or mouse use.

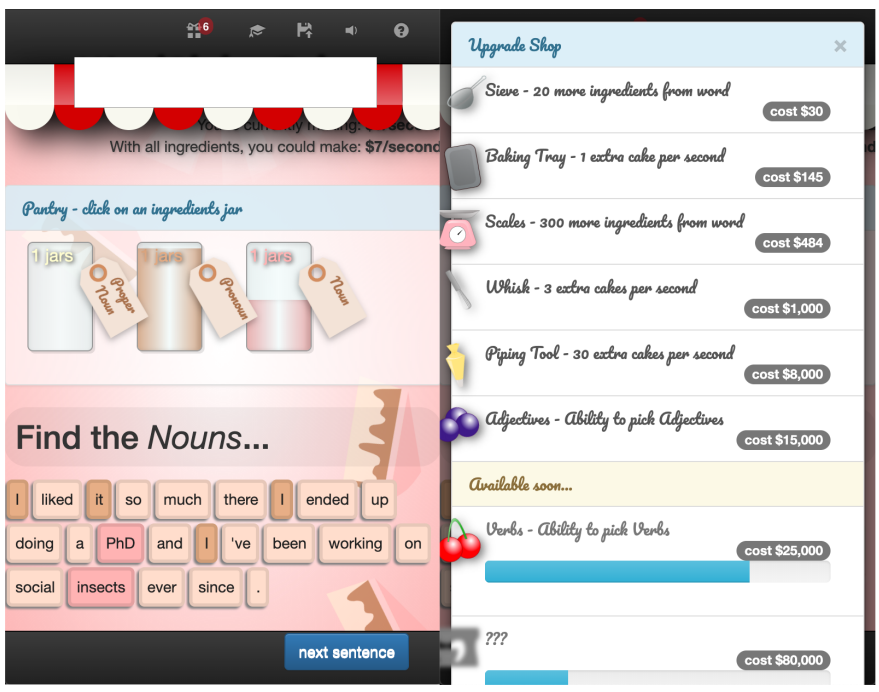

Fig. 4. WordClicker- Responsive Interface (Game and shop side by side)

The game is styled like a cake shop front with a red and white awning. The help system has a three dimensional menu theme (Figure 3).

Each label is associated with an ingredient. Cakes are displayed both in the background (falling) and in the foreground. These show the player which cake is currently being made from their available ingredients. All the combinations of cakes available are shown in Table I. In addition, colours are associated with the ingredients to show the player which ingredient jar they currently have selected. Selecting a jar changes the background colour of the screen to remind the player.

The ingredients displayed on the cake change depending on which ingredients the player has available at that time, as do the ingredients on the cakes falling in the background.

\section{E. Sound and Music}

A dissonant two note sound effect descending in pitch is given to feedback an incorrect action to the player. A harmonious sound increasing in pitch is used to feedback a correct action.

\section{ACKNOWLEDGEMENTS}

This research was supported in part by the EPSRC CDT in Intelligent Games and Game Intelligence (IGGI), EP/L015846/1; in part by the DALI project, ERC Grant 695662.

\section{REFERENCES}

[1] M. Lafourcade, A. Joubert, and N. Le Brun, Games With A Purpose (GWAPs). Wiley, 2015.

[2] M. Lafourcade, "Making people play for Lexical Acquisition with the JeuxDeMots prototype," p. 8, 2007.

[3] M. Poesio, J. Chamberlain, U. Kruschwitz, L. Robaldo, and L. Ducceschi, "Phrase detectives: Utilizing collective intelligence for internet-scale language resource creation," ACM Transactions on Interactive Intelligent Systems, vol. 3, no. 1, pp. 1-44, Apr. 2013. [Online] Available: http://dl.acm.org/citation.cfm?doid=2448116.2448119

[4] N. Venhuizen, K. Evang, V. Basile, and J. Bos, "Gamification for Word Sense Labeling," p. 7, 2013.

[5] D. Jurgens and R. Navigli, "Its All Fun and Games until Someone Annotates: Video Games with a Purpose for Linguistic Annotation," p. 16, 2014.

[6] D. Vannella, D. Jurgens, D. Scarfini, D. Toscani, and R. Navigli, "Validating and Extending Semantic Knowledge Bases using Video Games with a Purpose," in Proceedings of the 52nd Annual Meeting of the Association for Computational Linguistics (Volume 1: Long Papers). Baltimore, Maryland: Association for Computational Linguistics, 2014, pp. 1294-1304. [Online]. Available: http://aclweb.org/anthology/P141122 
[7] D. Dziedzic and W. Wodarczyk, "Making NLP games with a purpose fun to play using Free to Play mechanics: RoboCorp case study," p. 2, 2016.

[8] L. von Ahn and L. Dabbish, "Labeling images with a computer game," in Proceedings of the 2004 conference on Human factors in computing systems - CHI '04. Vienna, Austria: ACM Press, 2004, pp. 319-326. [Online]. Available: http://portal.acm.org/citation.cfm?doid=985692.985733

[9] S. Cooper, F. Khatib, A. Treuille, J. Barbero, J. Lee, M. Beenen, A. Leaver-Fay, D. Baker, Z. Popovi, and F. players, "Predicting protein structures with a multiplayer online game," Nature, vol. 466, no. 7307, pp. 756-760, Aug. 2010. [Online]. Available: http://www.nature.com/articles/nature09304

[10] L. von Ahn, "Games with a Purpose," Computer, vol. 39, no. 6, pp. 92-94, Jun. 2006. [Online]. Available: http://ieeexplore.iee.org/document/1642623/

[11] K. Tuite, "GWAPs: Games with a Problem," p. 7, 2014.

[12] J. Tanz, "The Curse of Cow Clicker: How a Cheeky Satire Became a Videogame Hit," Wired, vol. 20, no. 1, Dec. 2011. [Online]. Available: https://www.wired.com/2011/12/ff-cowclicker/

[13] S. Deterding, "Progress Wars: Idle Games and the Demarcation of Real Games," p. 2, 2016.

[14] J. Tanenbaum and J. Bizzocchi, "Rock Band: a case study in the design of embodied interface experience," in Proceedings of the 2009 ACM SIGGRAPH Symposium on Video Games - Sandbox '09. New Orleans, Louisiana: ACM Press, 2009, p. 127. [Online]. Available: http://portal.acm.org/citation.cfm?doid=1581073.1581093

[15] R. Kiberd, "Cookie Clicker, the Internets most pointlessly addictive game, is also its most subversive," Jan. 2016. [Online]. Available: https://kernelmag.dailydot.com/issue-sections/staffeditorials/15694/cookie-clicker-capitalist-dystopia/

[16] S. A. Alharthi, O. Alsaedi, Z. O. Toups, J. Tanenbaum, and J. Hammer, "Playing to Wait: A Taxonomy of Idle Games," in Proceedings of the 2018 CHI Conference on Human Factors in Computing Systems - CHI '18. Montreal QC, Canada: ACM Press, 2018, pp. 1-15. [Online]. Available: http://dl.acm.org/citation.cfm?doid=3173574.3174195 